\title{
High-Throughput Electron Diffraction Reveals a Hidden Novel Metal-Organic Framework for Electrocatalysis
}

\author{
Meng Ge, Zhehao Huang and Xiaodong Zou \\ Department of Materials and Environmental Chemistry, Stockholm University, Stockholm SE-106 91, Sweden \\ gemeng733@gmail.com
}

Metal-organic frameworks (MOFs) are known for their versatile combination of inorganic building units and organic linkers, which offers immense opportunities in a wide range of applications. However, many MOFs are typically synthesized as multiphasic polycrystalline powders, which are challenging for studies by X-ray diffraction. Therefore, developing new structural characterization techniques is highly desired in order to accelerate discoveries of new materials. Here, we report a high-throughput approach for structural analysis of MOF nano- and sub-microcrystals by three-dimensional electron diffraction (3DED). A new zeolitic-imidazolate framework (ZIF), denoted ZIF-EC1, was first discovered in a trace amount during the study of a known ZIF-CO -1 material by 3DED. The structures of both ZIFs were solved and refined using 3DED data. ZIF-EC1 has a dense 3D framework structure, which is built by linking mono- and bi-nuclear $\mathrm{Zn}$ clusters and 2-methylimidazolates $\left(\mathrm{mIm}^{-}\right)$. The discovery of this new MOF highlights the power of 3DED in developing new materials.

Keywords: Three-dimensional electron diffraction, continuous rotation electron diffraction, high throughput structural analysis, metal-organic frameworks 